\title{
Functional complementation between transcriptional methylation regulation and post-transcriptional microRNA regulation in the human genome
}

Zhixi Su, ${ }^{1,2+}$, Junfeng $\mathrm{Xia}^{1+}$, Zhongming Zhao ${ }^{1,3,4^{*}}$

From BIOCOMP 2010. The 2010 International Conference on Bioinformatics and Computational Biology Las Vegas, NV, USA. 12-15 July 2010

\begin{abstract}
Background: DNA methylation in the $5^{\prime}$ promoter regions of genes and microRNA (miRNA) regulation at the $3^{\prime}$ untranslated regions (UTRs) are two major epigenetic regulation mechanisms in most eukaryotes. Both DNA methylation and miRNA regulation can suppress gene expression and their corresponding protein product; thus, they play critical roles in cellular processes. Although there have been numerous investigations of gene regulation by methylation changes and miRNAs, there is no systematic genome-wide examination of their coordinated effects in any organism.

Results: In this study, we investigated the relationship between promoter methylation at the transcription level and miRNA regulation at the post-transcription level by taking advantage of recently released human methylome data and high quality miRNA and other gene annotation data. We found methylation level in the promoter regions and expression level was negatively correlated. Then, we showed that miRNAs tended to target the genes with a low DNA methylation level in their promoter regions. We further demonstrated that this observed pattern was not attributed to the gene expression level, expression broadness, or the number of transcription factor binding sites. Interestingly, we found miRNA target sites were significantly enriched in the genes located in differentially methylated regions or partially methylated domains. Finally, we explored the features of DNA methylation and miRNA regulation in cancer genes and found cancer genes tended to have low methylation level and more miRNA target sites.

Conclusion: This is the first genome-wide investigation of the combined regulation of gene expression. Our results supported a complementary regulation between DNA methylation (transcriptional level) and miRNA function (posttranscriptional level) in the human genome. The results were helpful for our understanding of the evolutionary forces towards organisms' complexity beyond traditional sequence level investigation.
\end{abstract}

\section{Background}

Epigenetics refers to the heritable changes that modify DNA or associated proteins without changing the DNA sequence itself [1]. It has been commonly accepted that both epigenetic mechanisms - DNA methylation

\footnotetext{
* Correspondence: zhongming.zhao@vanderbilt.edu.

+ Contributed equally

'Department of Biomedical Informatics, Vanderbilt University School of Medicine, Nashville, TN 37232, USA

Full list of author information is available at the end of the article
}

modification at the gene's promoter regions ( $5^{\prime}$ of the gene) and microRNA (miRNA) regulation at the 3' untranslated regions ( 3 ' UTRs) - are important in gene expression regulation. DNA methylation has been popularly investigated due to its heritable epigenetic modifications of the genome and has been implicated in the regulation of most cellular processes. These include embryonic development, transcription, chromatin structure, $\mathrm{X}$ chromosome inactivation, genomic imprinting and chromosome stability [2-6]. Aberrant DNA 
methylation has been frequently reported to influence gene expression and subsequently cause various human diseases, especially cancer [7-9]. The causal relationship between variation in promoter DNA methylation and difference in gene regulation has been well recognized $[10,11]$. Recent work [12] revealed that hypermethylation at promoter $\mathrm{CpG}$ sites typically results in a lower transcription level of downstream genes. When methylation was experimentally removed from a gene's promoter region, its transcription level would often be higher [13]. Among the $\sim 28$ million CpG dinucleotide sites that are susceptible to methylation in the human genome, approximately $10 \%$ are in the promoter regions of genes, in which they may physically obstruct the binding of transcriptional proteins to the gene or may be indirectly regulated by the recruitment of methyl-CpG-binding domain proteins through cytosine methylation [14-16]. The repression role in gene expression regulation by methylation modification in a gene's promoter region has been reinforced by current whole genome bisulfite sequencing of the methylomes of more than 20 eukaryotes [17].

miRNAs are a class of small noncoding RNA molecules that regulate eukaryotic gene expression at the post-transcriptional level. They specifically bind mRNAs in their 3' UTRs based on sequence complementation and lead to translational repression and gene silencing [18]. According to release 17 (April 26, 2011) of the miRNA database miRBase [19], there are 16,772 miRNA gene loci in 153 species and 19,724 distinct mature miRNA sequences [20]. Among them, the human genome encodes 1424 miRNA sequences, which may target approximately $60 \%$ of human protein-coding genes [21]. This huge number of miRNAs discovered so far indicates that many biological processes, including cell cycle control, cell growth and differentiation, apoptosis, and embryo development, are controlled by miRNAmediated gene expression regulation [22].

Although there have been many important advances in understanding gene silencing roles at the transcriptional level through DNA methylation modification and at the post-transcriptional level through miRNA regulation, it remains unclear how these two major mechanisms cooperate at the genome-wide level to influence cellular processes. Thus, a combinatory analysis of these two mechanisms is likely to reveal many important insights into a deeper understanding of gene regulation in cells. Considering that (1) DNA methylation acts on a gene's 5' promoter region, and transcription typically depends on demethylation of the promoter region, and (2) miRNAs target on 3' UTR to suppress gene's post-transcriptional activities, we hypothesized that there exists a functional complementation between transcriptional promoter region methylation regulation and post-transcriptional miRNA regulation. If this hypothesis is valid, we would infer that (1) miRNAs preferentially target genes with a low DNA methylation level at the promoter regions; (2) genes that are controlled by more miRNAs tend to have less promoter methylation regulation. We validated our hypothesis by deeply analyzing human methylome data in two cell lines. To the best of our knowledge, this is the first report of the complementary relationship between DNA methylation regulation and miRNA regulation in a eukaryotic genome. Furthermore, we found that cancer genes tended to be silenced by miRNAs and to escape from DNA methylation suppression, thereby supporting our hypothesis.

\section{Methods}

\section{Gene annotation}

Human and mouse gene structure data was retrieved from the Ensembl database (version 54), including the information of Ensembl gene ID, Ensembl transcript ID, transcript start (bp), transcript end (bp), Ensembl protein ID, 3' UTR start, 3' UTR end, chromosome, and strand. We extracted the promoter region and 3' UTR position information from Gene structure data. If there are multiple transcripts for a gene, the transcription start site (TSS) and 3' UTR of the major transcript were used [23]. We retained only those genes without distant alternative TSS ( $>200 \mathrm{bp}$ distance from the major TSS) and without ambiguous 3' UTR regions to avoid the potential inaccurate mapping of the gene expression data and gene structures.

\section{Analysis of DNA methylation data}

The single-base resolution DNA methylation data was retrieved from Lister et al. (2009) [15], including whole genome bisulfite sequencing data for two human cell lines: H1 human embryonic stem cells and IMR90 fetal lung fibroblasts. The methylation information for each promoter was extracted by mapping the promoter region (in a range of -1000 to $+200 \mathrm{bp}$ from the TSS) to the genome-wide methylation data from the H1/IMR90 cell line.

Based on single-base resolution bisulfite sequencing data, we used methylation broadness to measure the DNA methylation level in specific genome regions, which was calculated as the proportion of methylated CpG sites among the total CpG sites in a sequence (we denote it as "mCG/CG" hereafter).

We also used "normalized" CpG content, the observed over expected $\mathrm{CpG}$ ratio $\left(\mathrm{CpG}_{\mathrm{O} / \mathrm{E}}\right)$ in a sequence, to infer the pattern of DNA methylation in the human genome. $\mathrm{CpG}_{\mathrm{O} / \mathrm{E}}$ is a robust measure of the level of DNA methylation on an evolutionary time scale due to 
specific mutational mechanisms of methylated cytosines [23]. Briefly, methylated cytosines are hypermutable due to their vulnerability to spontaneous deamination, which causes a gradual depletion of $\mathrm{CpG}$ dinucleotides from methylated regions over evolutionary time. Consequently, genomic regions that are subject to strong germline DNA methylation (hypermethylated) would decrease the extent of $\mathrm{CpG}$ dinucleotide content over time and, thus, have lower-than-expected $\mathrm{CPG}_{\mathrm{O} / \mathrm{E}}$. In contrast, regions that undergo weak germline DNA methylation (hypomethylated) maintain high $\mathrm{CpG}_{\mathrm{O} / \mathrm{E}}$. This measure has been successfully used to indirectly measure historical DNA methylation levels. In particular, the pattern of DNA methylation inferred from $\mathrm{CpG}_{\mathrm{O} / \mathrm{E}}$ corresponds well to the actual pattern of DNA methylation in such diverse taxa as human and sea squirt. $\mathrm{CpG}_{\mathrm{O} / \mathrm{E}}$ was calculated as the frequency of $\mathrm{CpG}$ sites divided by the frequency of $\mathrm{C}$ and $\mathrm{G}$ [24]. The pattern of DNA methylation inferred from $\mathrm{Cp}_{\mathrm{O} / \mathrm{E}}$ corresponds well to the actual pattern of DNA methylation in human stem cells (H1 cell line) and fetal lung fibroblasts (IMR90) [14,15]. Since the DNA methylation level of two strands in any given genomics region are highly correlated, here we used the sense strand to represent the DNA methylation level for a given gene promoter region. Similar results were obtained in this study when we used the methylation level of anti-sense (data not shown).

\section{Compilation of miRNA targets}

The miRNAs and their predicted targets were extracted from $\mathrm{R}$ package RmiR.hsa [25], including miRNA target site prediction results from 6 sources: miRBase, targetScan, miRanda, tarBase, mirTarget 2 and PicTar. In this study, we used the target site prediction results from two approaches: mirTaeget 2 and PicTar.

\section{Analysis of human gene expression data}

We obtained the expression data of 409 microarray experiments from McVicker and Green (2010) [26], which were collected from 12 studies [12,13,27-36], representing a wide variety of germ and somatic tissues. As these studies used two different platforms (Affymetrix microarrays hgu133plus2 and hgu133A), we only considered the probe sets shared by both arrays. The methods to process the raw intensity data and to assign the probe sets to genes were described in McVicker and Green (2010) [26]. In total, we assigned an expression intensity of 9858 genes in 409 tissues. Among the 409 tissues, 64 containing germ cells were considered as germline tissues, with the exception of germ cell tumors, embryonic stem cells, and immortalized cell lines (see additional file 1).
Because the above data sets are highly redundant in terms of tissue or cell type, we only used Gene Expression Atlas data to estimate the relative expression broadness ( $E B$, number of tissues where a gene is expressed). This data has been widely used to estimate gene expression broadness. The Affymetrix raw data was downloaded from the website of the authors in reference [36]. It comprised 156 human (U133A/ GNF1H) microchip experiments in 79 tissues. The expression level detected by each probe set was obtained as the average difference (AD) value computed from MAS 5.0 algorithm (MAS5) [37]. The AD values were averaged among replicates. Using the annotation tables from the original study $[36,38]$ and the Ensembl EnsMart tool, we mapped the probe IDs used in the microarray experiments to Ensemble gene identifiers. In approximately $20 \%$ of the cases, multiple probes in the microarray targeted onto a single gene. The expression intensities of multiple probes that corresponded to one gene were averaged after discarding all the low-confidence probe sets (indicated by a suffix of "_x_at" or "_s_at" in the Affymetrix IDs) [39]. In this study, we used an AD value of 200 as the threshold to calculate the EB, as we did in our previous work [23].

The gene expression data of two human cell lines H1 and IMR90 was obtained from reference [15]. The expression data was generated by a whole RNA sequencing (RNA-Seq) approach. The reads per kilobase of transcript per million reads (RPKM) were used to represent the expression level of each gene.

\section{Cancer genes}

We retrieved 427 human cancer genes and their annotations from the Cancer Gene Census database (CGC, 2010-03-30 version) [40]. Since a cancer gene may act in a dominant or recessive manner [41,42], we classified these 427 cancer genes as two groups, i.e., dominant gene group (337 genes) and recessive gene group (85 genes), according to their annotations in the CGC database. There were 5 genes with ambiguous classification in the database and they were excluded in this analysis.

\section{Human-specific insertion/deletion (indel) events in 3' UTRs}

We identified the human-specific indel events in 3' UTR regions as described in [43]. The 17-way vertebrate alignment, i.e., multiple alignments of 16 vertebrate genomes to the human genome (hg18), was obtained from [44].

An in-house Perl script was used to extract the orthologous 3' UTR alignment information and to identify the human-specific indel events. Human-specific insertion event rate and deletion event rate in the 3' UTR 
regions were calculated based on percent nucleotide difference. The indel rate equals to the sum of the lengths of all indels in the aligned human sequences divided by the total length of the aligned sequences.

\section{Results and discussion}

Correlation between gene expression level and promoter DNA methylation

Although methylation of gene's promoter regions has long been considered a suppressor of gene expression $[17,45]$, it still remains unclear to which extent the promoter's DNA methylation contributes to the influence of gene expression level $[45,46]$. For example, most promoters having $\mathrm{CpG}$ islands (CGIs) remain unmethylated even in cells that do not express the corresponding gene. On the contrary, most CpG-poor promoters are hypermethylated even in somatic cells where the genes are expressed [47]. What is equally uncertain is the contribution of promoter methylation to the tissue-specific gene expression. Although many studies have shown the tissue-specific differentially methylated regions ( $\mathrm{T}$ DMRs) could connect to the gene expression reprogramming in different tissues or developmental stages, others failed to demonstrate such a connection based on the analysis of a small set of genes $[48,49]$.To better understand the relationship between DNA methylation regulation and the gene expression regulation through miRNA targeting, we explored to what extent promoter methylation affects the gene expression level using the genome-wide data set collected in this study. We used two independent measurements, i.e., methylation broadness and normalized $\mathrm{CpG}$ content $\left(\mathrm{CpG}_{\mathrm{O} / \mathrm{E}}\right)$, to test the correlation of promoter methylation and gene expression level.

First, we calculated the broadness of DNA methylation in each gene promoter region in human $\mathrm{H} 1$ embryonic stem cells and IMR90 fetal lung fibroblasts, based on the recently published whole genome single-base resolution methylome data [15]. Methylation broadness measures the fraction of cytosine sites detected as methylated in a given DNA segment, which is calculated as the proportion of methylated sites over the total sites in a sequence (termed as mCG/CG) [17]. We calculated the pairwise correlation between promoter DNA methylation and gene expression level. We found gene expression intensity was significantly and negatively correlated with the methylation level in the promoter regions, both in H1 cells $\left(\rho=-0.468, P<10^{-15}\right)$ and in IMR90 cells $(\rho$ $\left.=-0.473, P<10^{-15}\right)$. Next, we used $\mathrm{CpG}_{\mathrm{O} / \mathrm{E}}$ to approximately infer the pattern of DNA methylation in the human genome. As a robust measurement of the level of germline DNA methylation on an evolutionary time scale [24], low $\mathrm{CpG}_{\mathrm{O} / \mathrm{E}}$ and high $\mathrm{CpG}_{\mathrm{O} / \mathrm{E}}$ reflect hypermethylation and hypomethylation, respectively. We calculated the correlation between $\mathrm{Cp}_{\mathrm{O} / \mathrm{E}}$ and gene expression level for a wide range of tissues. As shown in Figure 1, gene expression in most germline tissues was positively correlated with $\mathrm{CpG}_{\mathrm{O} / \mathrm{E}}$. Remarkably, we found the correlation is more significant in female germline tissues than in male germline tissues. The average gene expression intensity in all germline tissues is also significantly correlated with promoter $\mathrm{CPG}_{\mathrm{O} / \mathrm{E}}(\rho$ $=0.37, P<10^{-15}$ ). Our results also showed either weak correlation or even no significant correlation among most somatic tissues (Figure 1). In summary, using different DNA methylation measurements, we found methylation level in a gene's promoter regions was negatively correlated with expression level at the whole genome level. It is worth noting that we found a more significant correlation between gene promoter DNA methylation level and gene expression level than the previous studies $[3,15]$. One possible reason is that we only used the genes with unique TSS or largely overlapping promoter regions (see Methods).

\section{miRNAs preferentially target the genes with low DNA methylation level at the promoter regions}

We next tested the hypothesis that a functional complementation exists between transcriptional promoter region methylation regulation and post-transcriptional microRNA regulation. We retrieved unique miRNAs and their target sites for each human gene based on the predicted miRNA binding sites using mirTarget2 [50] and PicTar [51] algorithms. We chose these two

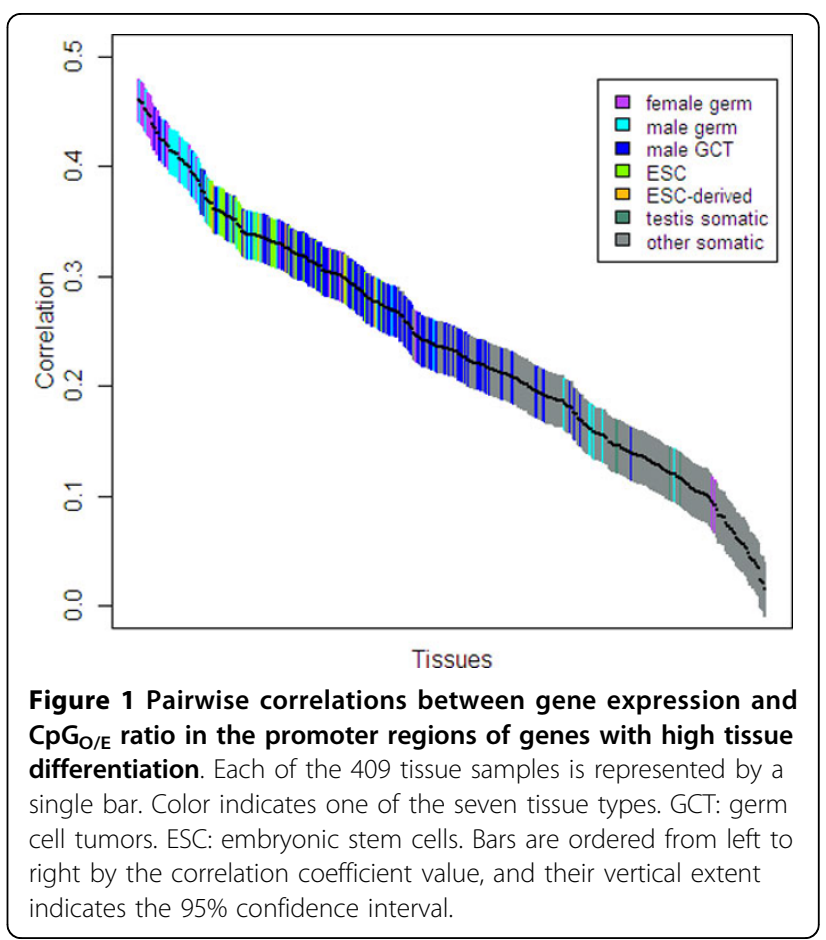


algorithms because most of the randomly selected miRNA targets predicted by mirTarget 2 and PicTar have been validated as true targets [50,52]. Genes that have long 3' UTRs are likely to be regulated by more miRNAs [53]; thus, we treated the 3' UTR length as a proxy of the number of miRNA target sites for an additional correlation analysis.

There were 12,730 genes that had both miRNA target prediction by mirTarget 2 and promoter methylation measured using human $\mathrm{H} 1$ cells. Using this dataset, we found a significant negative correlation between gene promoter methylation and number of miRNA target sites (Spearman's $\rho=-0.29, P<10^{-15}$ ) (Table 1, Figure 2). Similarly, we found a significant negative correlation between gene promoter methylation and number of miRNA target sites $\left(\rho=-0.26, P<10^{-15}\right)$ based on the 12,731 genes having both miRNA target prediction by mirTarget2 and promoter methylation from methylome of human IMR90 cells (Table 1, Figure 2). Moreover, using the $\mathrm{CPG}_{\mathrm{O} / \mathrm{E}}$ value in the promoter regions as a proxy of the promoter methylation level in germline cells, we found a significant positive correlation between $\mathrm{CpG}_{\mathrm{O} / \mathrm{E}}$ and the number of miRNA target sites $(\rho=$ $0.29, P<10^{-15}$ ) (Table 1, Figure 3). This positive correlation between $\mathrm{CPG}_{\mathrm{O} / \mathrm{E}}$ and the number of miRNA target sites is consistent with the negative correlations above, because $\mathrm{CPG}_{\mathrm{O} / \mathrm{E}}$ reversely reflects the promoter methylation level. Finally, when we used the miRNA target site data predicted by PicTar, we had very similar results (Table 1), indicating our findings are reliable.

We further used the 3' UTR length to approximately measure the number of miRNA target sites. Consistent with the above results, we found negative correlations between 3' UTR length and promoter methylation level in both human methylomes (H1 and IMR90) (Table 1). This analysis revealed that the genes with a higher promoter methylation level tended to have shorter 3' UTRs at the genome level.
We questioned whether the observed correlations above are unique in the human genome. Thus, we investigated the relationship between promoter DNA methylation level and the number of miRNA target sites in mice. We retrieved the corresponding gene structure data from the ENSEMBL database. The data processes that included the definition of TSS and estimation of 3' UTR length were the same as in humans, as described in the Methods section. We found a highly significant correlation between promoter $\mathrm{CpG}_{\mathrm{O} / \mathrm{E}}$ and 3' UTR length (Spearman's $\rho=0.24 ; P<10^{-15}$ ), indicating that the negative correlation pattern between promoter region methylation and number of miRNA target sites still holds in mice. Since mammalian genomes share many CpG island features in their promoter regions [4], it is likely that the observed correlation is common in mammals, or even in many vertebrates.

\section{Enrichment of miRNA targets among genes with lower promoter methylation level is not a by-product of gene expression level, expression broadness or the number of transcription factor binding sites}

We next specifically investigated whether the above observed enrichment of miRNA targets among genes with a lower promoter methylation level was a by-product of ancillary features of the analyzed gene sets. The results from the following analyses indicated this was not the by-product.

First, we asked whether the relationship between DNA methylation and miRNA regulation could be explained by the underlying gene expression levels since the DNA methylation of a gene's promoter regions and gene expression level is correlated in the majority of eukaryotes, and gene expression level is often positively correlated with the number of miRNA target sites. We estimated partial correlations [54] between DNA methylation and number of miRNA target sites after removing the contributions of gene expression level. The

Table 1 Spearman's rank correlation coefficients ( $\rho$ s) and partial correlations between gene's promoter methylation level and the number of microRNA target sites

\begin{tabular}{|c|c|c|c|c|c|c|}
\hline \multirow[b]{2}{*}{$\begin{array}{l}\text { Promoter } \\
\text { methylation } \\
\text { level }(m)\end{array}$} & \multicolumn{2}{|c|}{$\begin{array}{l}\text { Number of microRNA target sites by } \\
\text { mirTarget2 (Nt) }\end{array}$} & \multicolumn{2}{|c|}{$\begin{array}{l}\text { Number of microRNA target sites by } \\
\text { PicTar }(N p)\end{array}$} & \multicolumn{2}{|c|}{ 3' UTR length (UL) } \\
\hline & $\begin{array}{l}\text { Correlation } \\
\rho_{\mathrm{s}}(m, N t)\end{array}$ & $\begin{array}{l}\text { Partial correlation } \rho_{s}(m, \\
N t \mid g e n e \text { expression level) }\end{array}$ & $\begin{array}{l}\text { Correlation } \\
\rho_{\mathrm{s}}(m, N p)\end{array}$ & $\begin{array}{l}\text { Partial correlation } \rho s(m, \\
N p \mid g e n e \text { expression level) }\end{array}$ & $\begin{array}{l}\text { Correlation } \\
\rho_{\mathrm{s}}(m, U L)\end{array}$ & $\begin{array}{l}\text { Partial correlation } \rho_{s}(m, \\
U L \mid \text { gene expression level) }\end{array}$ \\
\hline $\begin{array}{l}\text { Promoter mCG/ } \\
\text { CG } \\
(\mathrm{H} 1)\end{array}$ & $-0.29 * * *$ & $-0.19^{* * *}$ & $-0.29^{* * *}$ & $-0.22^{* * *}$ & $-0.31^{* * *}$ & $-0.22^{* * *}$ \\
\hline $\begin{array}{l}\text { Promoter mCG/ } \\
\text { CG (IMR90) }\end{array}$ & $-0.26^{* * *}$ & $-0.19^{* * *}$ & $-0.28^{* * *}$ & $-0.19^{* * *}$ & $-0.29^{* * *}$ & $-0.20^{* * *}$ \\
\hline Promoter $\mathrm{CpG}_{\mathrm{O} / \mathrm{E}}$ & $0.29 * * *$ & $0.16^{* * * \dagger}$ & $0.28^{* * *}$ & $0.18 * *{ }^{\dagger}$ & $0.31^{* * *}$ & $0.19 * * *^{\dagger}$ \\
\hline
\end{tabular}

${ }^{\dagger}$ A germline tissue (FETAL.OVARY.14.4WK) having the most significant correlation between expression level and promoter region $\mathrm{CpG}_{\mathrm{O} / \mathrm{E}}$ ratio was selected [30]. *** $P<10^{-15}$. 


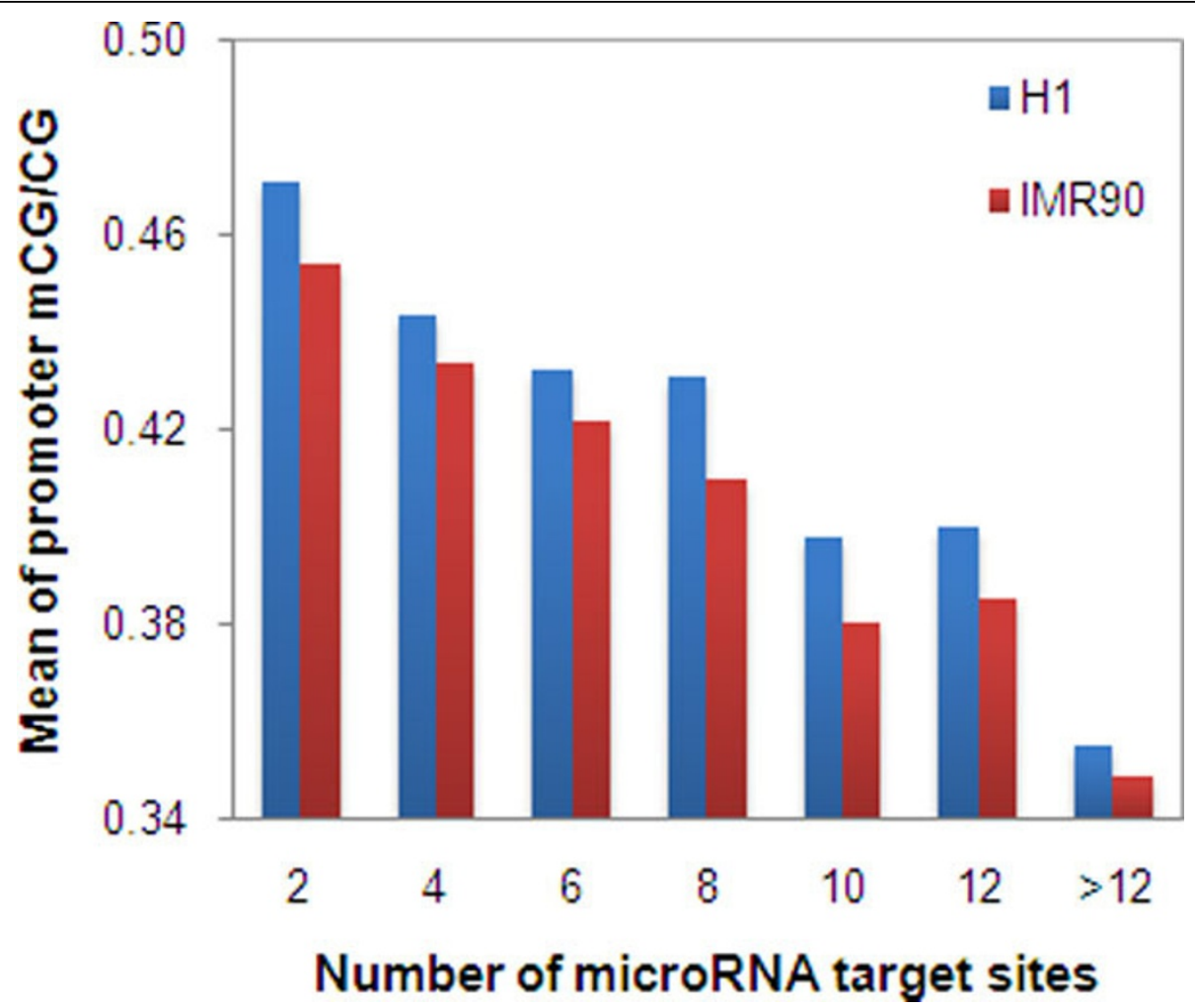

Figure 2 The correlation between methylation level in promoter regions and number of microRNA target sites. The number of microRNA target sites in each gene was predicted by mirTarget2. The methylation data was from the methylomes at base resolution of two human cells [15].

corresponding corrections were still highly significant, suggesting that covariance between DNA methylation (or the number of miRNA target sites) and gene expression level could not account for the observed relationships between DNA methylation and the number of miRNA target sites. As shown in Table 1. Although the partial correlations between DNA methylation and miRNA regulation decreased after removing the effects of gene expression level, they still showed high significance

Second, broadly expressed genes tended to avoid miRNA regulation $[55,56]$, implying that the correlation between promoter methylation and miRNA regulation could have been affected by the greater chance of higher DNA methylation level in broadly expressed genes' promoter regions.

We indeed found the promoter methylation level was negatively correlated with gene expression broadness (EB) (for mCG/CG using H1 methylome data, Spearman's $\rho=-0.19, P<10^{-15}$; for $\mathrm{CpG}_{\mathrm{O} / \mathrm{E}}, \rho=0.22, P<10^{-}$

${ }^{15}$ ) (Figure 4a). However, no significant correlation between the number of miRNA target sites and EB was observed (for miRNA target sites based on MirTarget2, $\rho=-0.003, P=>0.1$ ) (Figure $4 \mathrm{~b}$ ), and only a very weak correlation between the length of UTRs and EB ( $\rho=$ $0.03, P=0.002$ ) was observed. We had similar results using the methylation data of IMR90 and/or using the predicted miRNA target sites by PicTar (data not shown). Therefore, the effect of EB on the correlation of promoter methylation level and miRNA target sites could be largely ruled out.

Third, recent studies found genes with more transcription factor binding sites (TFBS) have a higher probability to be controlled by miRNAs [57]. We examined whether the promoter methylation levels are correlated with the number of TFBS. We extracted the TFBS data from [58]. A total of 22,067 genes had both TFBS and promoter methylation data. We found the correlation between TFBS and promoter methylation was very weak (Spearman's $\rho=-0.016$ for TFBS and $\mathrm{CpG}_{\mathrm{OE}} ; \rho=-0.07$ for TFBS and mCG/CG using H1 mythylome data). This observation suggested that the correlations between promoter methylation level and the number of miRNA targets was not a side effect of the correlation of TFBS site number and the number of miRNA target sites.

Finally, a previous study found that gene evolutionary rates were negatively correlated with the number of their regulatory miRNAs [53]. Therefore, we speculated 


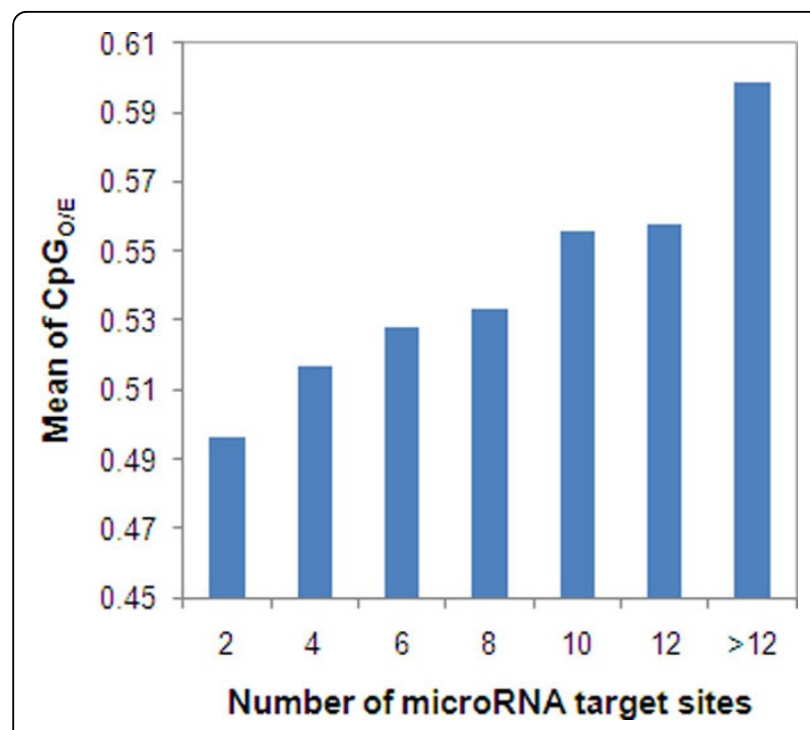

Figure 3 The correlation between $\mathrm{CpG}_{\mathrm{O} / \mathrm{E}}$ ratio in promoter regions and number of microRNA target sites. The number of miRNA target sites in each gene was predicted by mirTarget2.

genes with stronger promoter methylation repression (tend to be regulated by fewer miRNAs) might have evolved faster in their 3' UTRs and could have insertion or deletion bias. A possible mechanism of the negative correlation between promoter methylation and the number of regulatory miRNAs is that genes with hypermethylated promoters may in turn shorten their 3' UTRs to reduce possible miRNA regulation. We tested this hypothesis by the following analyses. We extracted the human-mouse one-to-one orthologous 3' UTR sequences from PACdb [59] and aligned these orthologous sequences using the computer program Clustal W [60]. We calculated the substitution rates per site (termed as $K_{3 u}$ ) based on the Kimura's two-parameter model [61]. We found a weak positive correlation between $K_{3 u}$ and the promoter methylation level (Spearman's $\rho=0.15, P<10^{-15}$ between $K_{3 u}$ and mCG/CG using $\mathrm{H} 1$ mythylome data; $\rho=-0.1, P<10^{-10}$ between $K_{3 u}$ and $\mathrm{CpG}_{\mathrm{O} / \mathrm{E}}$ ), indicating promoter hypermethylated genes tended to evolve faster in their 3' UTRs. We identified the human-specific insertion rate and deletion rate for the 3' UTRs of all genes (see Methods). However, there was no evidence to show that promoter hypermethylated genes tended to shorten their 3' UTR length $(P>0.1)$. Further studies of promoter methylation and 3 ' UTR evolution will be needed to uncover the underlying mechanisms of the connection between promoter methylation level and the number of miRNA target sites.

miRNA targets are significantly enriched in genes located in differentially methylated regions or partially methylated domains

Some genes may belong to a specific group of genes that are preferentially regulated by miRNAs or promoter region methylation. It is interesting to investigate the functional complementation between transcriptional promoter methylation and post-transcriptional miRNA regulation in such groups of genes. Specifically, we identified the genes located in differentially methylated regions (DMRs) and partially methylated domains (PMDs) using the data from Lister et al. [15]. According to Lister et al. [15], the DMRs were identified as the regions of the genome enriched for sites of higher levels of DNA methylation in IMR90 relative to $\mathrm{H} 1$ by Fisher's
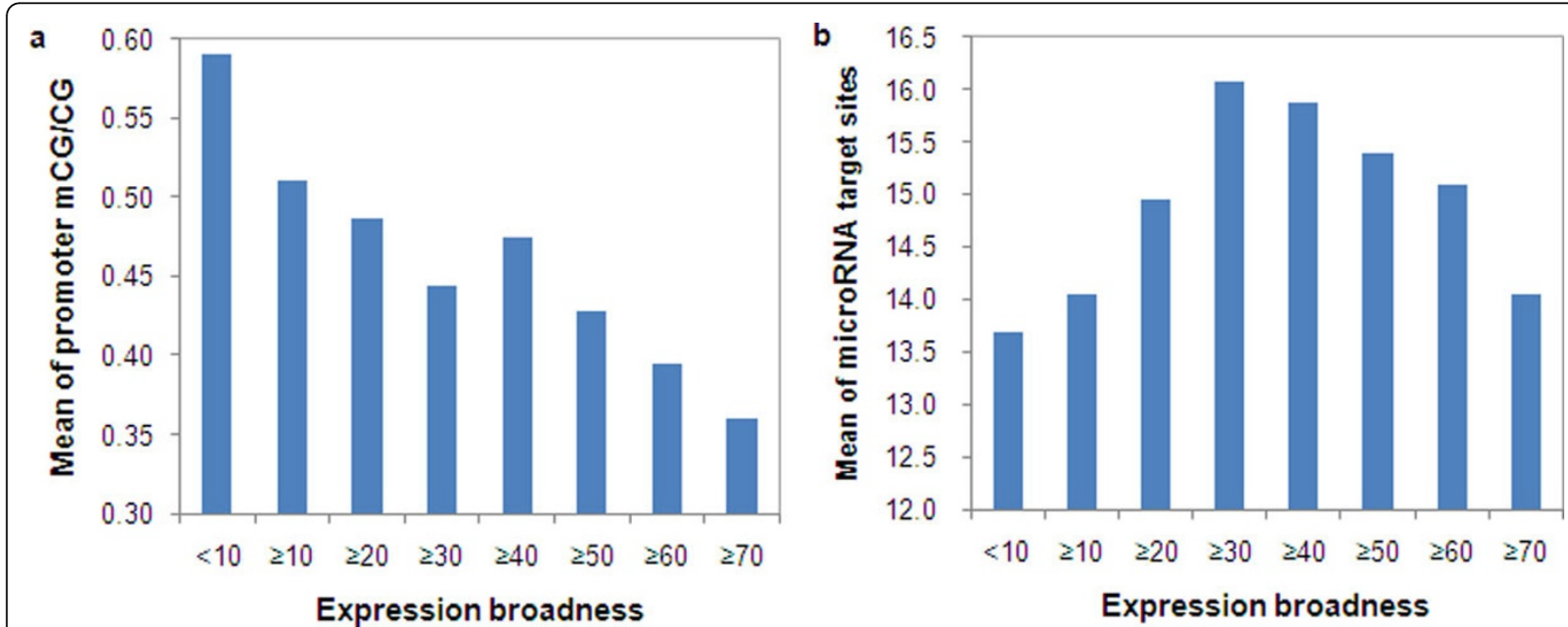

Figure 4 Distribution of promoter methylation level and number of microRNA target sites in genes by their expression broadness. (a) Negative correlation between promoter methylation level (mCG/CG) in human cell H1 and expression broadness. (b) microRNAs preferentially target the genes with intermediate expression broadness. 

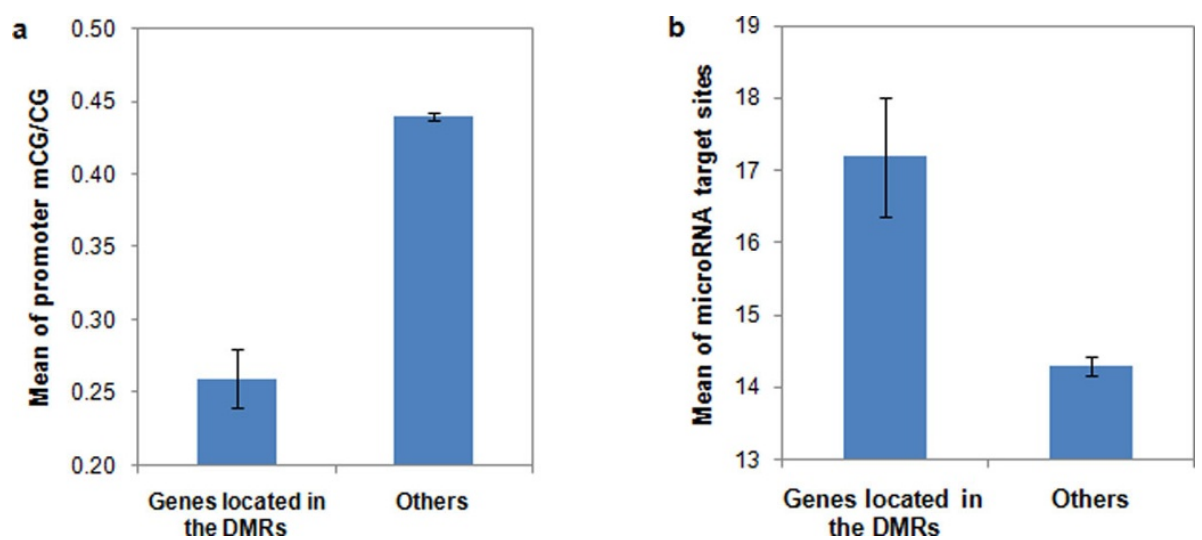

Figure 5 Genes located in differentially methylated regions (DMRs) tend to have low methylation level measured by promoter mCG/ CG and more microRNA target sites. Error bar: standard error.

Exact Test. There were 491 regions considered as DMRs using the methylome data from H1 and IMR90 cell lines. For the genes located at either DMRs or other genomic regions, we calculated the average number of miRNA target sites and average value of promoter methylation level, respectively. Using the $\mathrm{H} 1$ methylome data, on average, genes located at the DMRs and other regions had mCG/CG ratios of 0.26 and $0.44\left(P<10^{-15}\right.$, Mann-Whitney U test) (Figure 5a), and 17.2 and 14.3 miRNA targets sites $\left(P<10^{-6}\right.$, Mann-Whitney $U$ test $)$ (Figure $5 \mathrm{~b}$ ), respectively. These findings indicate that genes located in DMRs tended to maintain a low methylation level, whereas they might be regulated by more miRNAs. Therefore, there exists a negative correlation between DNA methylation level and the number of miRNA target sites.

Lister et al. showed a trend of decreased level of methylation level in PMDs (partially methylated domains in IMR90 cell line, contiguous regions with an average methylation level less than 70\%). We calculated the average number of miRNA target sites in PMDs and other genomic regions. As expected, genes located in PMDs had a lower promoter methylation level $\left(P<10^{-}\right.$ $\left.{ }^{4}\right)$ and were regulated by more miRNAs $\left(P<10^{-6}\right)$ (Figure 6$)$. This result again demonstrated a negative correlation existed between promoter methylation level and the number of miRNA target sites.

\section{DNA methylation and miRNA regulation in cancer genes}

Cancer is a common complex disease, and many genes have been reported as involved in the development of cancer. Since cancer genes have been extensively studied and often found to be regulated by miRNAs, it is interesting to examine whether the cancer genes are more likely to have low methylation in accordance with our hypothesis and our observations above. To test this hypothesis, we retrieved human cancer genes and their annotations from the CGC database and compared the
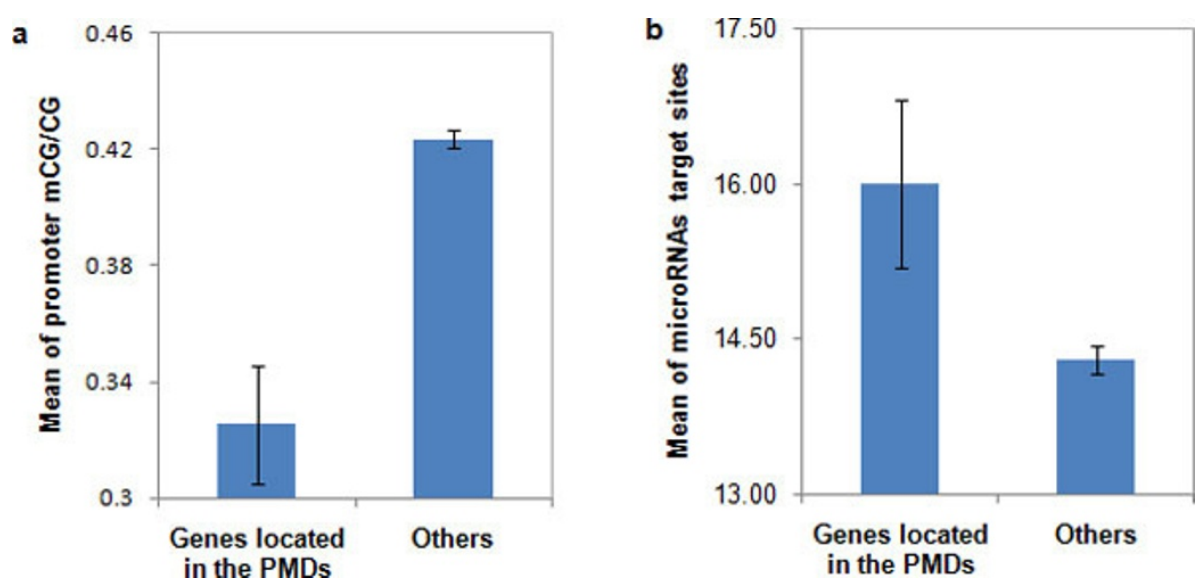

Figure 6 Genes located in partially methylated domains (PMDs) tend to have low methylation level measured by promoter mCG/CG and more microRNA target sites. Error bar: standard error. 
Table 2 Summary of microRNA target sites and methylation data in gene's promoter regions

\begin{tabular}{lllll}
\hline $\begin{array}{l}\text { Gene } \\
\text { categories }\end{array}$ & $\begin{array}{l}\text { Mean of microRNA target sites } \\
\text { (gene number) }\end{array}$ & $\begin{array}{l}\text { Mean of } \mathbf{m C G} / \mathrm{CG} \text { (gene } \\
\text { number*) }\end{array}$ & $\begin{array}{l}\text { Mean of } \text { CpG }_{\mathrm{O} / \mathrm{E}} \text { (gene } \\
\text { number) }\end{array}$ & $\begin{array}{l}\text { CGI number in promoter region } \\
\text { (gene number) }\end{array}$ \\
\hline $\begin{array}{l}\text { Cancer } \\
\text { genes }\end{array}$ & $\mathbf{1 8 . 6 0}(379)$ & $\mathbf{0 . 3 3}(381)$ & $\mathbf{0 . 6 2 ( 3 8 1 )}$ & $\mathbf{0 . 7 6}(412)$ \\
Dominant & $19.18(302)$ & $0.33(299)$ & $0.61(299)$ & $0.73(327)$ \\
Recessive & $16.16(76)$ & $0.32(80)$ & $0.66(80)$ & $\mathbf{0 . 8 7}(84)$ \\
Others & $\mathbf{1 4 . 3 4}(14,315)$ & $\mathbf{0 . 5 3}(20,359)$ & $\mathbf{0 . 4 8}(20,359)$ & $\mathbf{0 . 5 3}(21,767)$ \\
\hline
\end{tabular}

CGI: CpG island. Dominant and Recessive genes are two major cancer gene categories.

*Those genes with $\mathrm{CpG}$ reads in their promoter region less than 3 were excluded.

cancer genes and other genes by their numbers of miRNA target sites, normalized methylation level, $\mathrm{CpG}_{\mathrm{O} /}$ $\mathrm{E}$ and number of CGIs in the promoter regions. Table 2 summarizes the results of these analyses. We found that cancer genes tended to have more miRNA target sites than other genes (average 18.60 miRNA target sites for cancer genes versus 14.34 for other genes, $P<10^{-15}$, Mann-Whitney U-test). On the contrary, cancer genes had lower methylation levels than other genes, regardless of whether the methylation level was measured by methylation broadness (mCG/CG), normalized CpG content $\left(\mathrm{CpG}_{\mathrm{O} / \mathrm{E}}\right)$, or number of CGIs in the promoter regions (Table 2). For example, the normalized methylation level in cancer genes' promoter regions was lower than other genes (average 0.33 for cancer genes versus 0.53 for other genes, $P<10^{-15}$, Mann-Whitney U-test).

We next compared the features in two major groups of cancer genes: dominant and recessive cancer genes. Among the 427 cancer genes, there were 337 dominant cancer genes and 85 recessive cancer genes based on their annotations in the CGC database. We analyzed their DNA methylation levels and number of miRNA target sites. For a normalized methylation level and $\mathrm{Cp}_{\mathrm{O} / \mathrm{E}}$, no significant difference was detected between the dominant and recessive cancer genes. However, the number of miRNA target sites in the dominant cancer genes (19.18) was larger than that of recessive cancer genes (16.16). Finally, the number of CGIs in the promoter regions of the dominant cancer genes (0.73) was significantly smaller than that of the recessive cancer genes $\left(0.87, \chi^{2}\right.$ test, $\left.P<10^{-15}\right)$. These comparisons suggested the different inheritable mechanisms of the dominant and recessive cancer genes in cancer, as we recently examined in the protein-protein interaction level [62].

Collectively, we observed that the promoter region methylation level in cancer genes was negatively correlated with their number of miRNA target sites. This observation still held after filtering the potential confounding effects from gene expression level or expression broadness. This analysis indicated that the cancer genes tended to be silenced by miRNA genes but could escape from DNA methylation suppression.

\section{Conclusion}

To understand how DNA methylation and miRNA regulate the expression of their target genes, many previous exploratory studies have been reported, but all of them focused on the effect of each mechanism on the expression of target genes. In this study, we investigated the relationship between promoter methylation and miRNA regulation at the genome level by taking advantage of recently released human methylome data and high quality miRNA and other gene annotation data. Our results suggested that there is a functional complementation between promoter methylation regulation at the transcription level and miRNA regulation at the post-transcriptional level. Specifically, the genes that are under stronger promoter DNA methylation control tend to avoid miRNA regulation by having fewer miRNA target sites, and vice versa.

From an evolutionary perspective, both recruitment of DNA methylation in a gene's promoter region and the advent of new miRNA genes during the transition from invertebrate to vertebrate contributed to the high complexity of vertebrate organisms and cell types [63-65]. Although many recent studies have greatly improved our understanding of the evolutionary adaptations and conservation of DNA methylation and miRNA regulation, the relationship between DNA methylation and miRNA regulation, and how these two mechanisms dynamically influence each other's evolution and function, remain poorly understood. The results supporting complementary regulation between DNA methylation and miRNA function in this study provided the first attempt to uncover such an important and complex regulation system, which will help us understand the evolutionary forces towards organisms' complexity beyond traditional sequence level investigation.

\section{Additional material}

Additional file 1: The gene expression intensities in germline tissues. Totally 6569 genes can be assigned the expression intensities in 64 tissues. CPGo/E was calculated for the promoter region (-1000 bp to +200 bp relative to the TSS) of each gene. 


\section{Acknowledgements}

We thank Graham McVicker and Phil Green (University of Washington) for their help in microarray data analysis and providing their processed data. We thank members of the Zhao lab for useful discussion and suggestions and Rebecca Posey for English polishing of the manuscript. This work was partially supported by NIH grant (LM009598) from the National Library of Medicine. Z. Zhao received additional support from Vanderbilt's Specialized Program of Research Excellence in Gl Cancer grant (50CA95103) and the VICC Cancer Center Core grant (P30-CA68485).

\section{Author details}

'Department of Biomedical Informatics, Vanderbilt University School of Medicine, Nashville, TN 37232, USA. ${ }^{2}$ MOE Key Laboratory of Contemporary Anthropology and Center for Evolutionary Biology, School of Life Sciences, Fudan University, Shanghai 200433, China. ${ }^{3}$ Department of Psychiatry, Vanderbilt University School of Medicine, Nashville, TN 37232, USA ${ }^{4}$ Department of Cancer Biology, Vanderbilt University School of Medicine, Nashville, TN 37232, USA.

\section{Authors' contributions}

ZS designed the study, carried out the data analysis and drafted the manuscript. JX participated in the data analysis and drafted the manuscript. ZZ conceived of the study, designed the project, participated in the data analysis and drafted the manuscript. All authors read and approved the final manuscript.

\section{Competing interests}

The authors declare that they have no competing interests.

Published: 23 December 2011

\section{References}

1. Egger G, Liang G, Aparicio A, Jones PA: Epigenetics in human disease and prospects for epigenetic therapy. Nature 2004, 429:457-463.

2. Lippman Z, Gendrel AV, Black M, Vaughn MW, Dedhia N, McCombie WR, Lavine K, Mittal V, May B, Kasschau KD, et al: Role of transposable elements in heterochromatin and epigenetic control. Nature 2004, 430:471-476

3. Weber M, Hellmann I, Stadler MB, Ramos L, Paabo S, Rebhan M, Schubeler D: Distribution, silencing potential and evolutionary impact of promoter DNA methylation in the human genome. Nat Genet 2007, 39:457-466.

4. Han L, Su B, Li W-H, Zhao Z: CpG island density and its correlations with genomic features in mammalian genomes. Genome Biol 2008, 9:R79.

5. Han L, Zhao Z: Contrast features of CpG islands in the promoter and other regions in the dog genome. Genomics 2009, 94:117-124.

6. Zhao Z, Han L: CpG islands: algorithms and applications in methylation studies. Biochem Biophys Res Commun 2009, 382:643-645.

7. Robertson KD: DNA methylation and human disease. Nat Rev Genet 2005, 6:597-610.

8. Szyf M: The role of DNA hypermethylation and demethylation in cance and cancer therapy. Curr Oncol 2008, 15:72-75.

9. Szyf M, Pakneshan P, Rabbani SA: DNA methylation and breast cancer. Biochem Pharmacol 2004, 68:1187-1197.

10. Jaenisch R, Bird A: Epigenetic regulation of gene expression: how the genome integrates intrinsic and environmental signals. Nat Genet 2003, 33:245-254.

11. Murrell A, Rakyan VK, Beck S: From genome to epigenome. Hum Mol Genet 2005, 14:R3-R10

12. Perez-Iratxeta C, Palidwor G, Porter CJ, Sanche NA, Huska MR, Suomela BP, Muro EM, Krzyzanowski PM, Hughes E, Campbell PA: Study of stem cell function using microarray experiments. FEBS Lett 2005, 579:1795-1801.

13. Wu X, Schmidt JA, Avarbock MR, Tobias JW, Carlson CA, Kolon TF, Ginsberg JP, Brinster RL: Prepubertal human spermatogonia and mouse gonocytes share conserved gene expression of germline stem cell regulatory molecules. Proc Natl Acad Sci USA 2009, 106:21672-21677.

14. Li Y, Zhu J, Tian G, Li N, Li Q, Ye M, Zheng H, Yu J, Wu H, Sun J, et al: The DNA methylome of human peripheral blood mononuclear cells. PLOS Biol 2010, 8(11):e1000533.

15. Lister R, Pelizzola M, Dowen RH, Hawkins RD, Hon G, Tonti-Filippini J, Nery JR, Lee L, Ye Z, Ngo QM, et al: Human DNA methylomes at base resolution show widespread epigenomic differences. Nature 2009 462:315-322

16. Lister R, Pelizzola M, Kida YS, Hawkins RD, Nery JR, Hon G, AntosiewiczBourget J, O'Malley R, Castanon R, Klugman S, et al: Hotspots of aberrant epigenomic reprogramming in human induced pluripotent stem cells. Nature 2011, 471(7336):68-73

17. Su Z, Han L, Zhao Z: Conservation and divergence of DNA methylation in eukaryotes: New insights from single base-resolution DNA methylomes. Epigenetics 2011, 6:134-140.

18. Filipowicz W, Bhattacharyya SN, Sonenberg N: Mechanisms of posttranscriptional regulation by microRNAs: are the answers in sight? Nat Rev Genet 2008, 9:102-114.

19. Kozomara A, Griffiths-Jones S: miRBase: integrating microRNA annotation and deep-sequencing data. Nucleic Acids Res 2011, 39:D152-D157.

20. Griffiths-Jones S, Saini HK, Van Dongen S, Enright AJ: miRBase: tools for microRNA genomics. Nucleic Acids Res 2008, 36:D154-D158.

21. Friedman $L M$, Dror AA, Mor E, Tenne $T$, Toren $G$, Satoh $T$, Biesemeier DJ, Shomron N, Fekete DM, Hornstein E, Avraham KB: MicroRNAs are essential for development and function of inner ear hair cells in vertebrates. ProC Natl Acad Sci USA 2009, 106:7915-7920.

22. Bushati N, Cohen SM: microRNA functions. Annu Rev Cell Dev Biol 2007, 23:175-205.

23. Jiang C, Han L, Su B, Li WH, Zhao Z: Features and trend of loss of promoter-associated CpG islands in the human and mouse genomes. Mol Biol Evol 2007, 24:1991-2000.

24. Elango N, Hunt BG, Goodisman MA, Yi SV: DNA methylation is widespread and associated with differential gene expression in castes of the honeybee, Apis mellifera. Proc Natl Acad Sci USA 2009, 106:11206-11211.

25. R package RmiR.hsa. [http://www.bioconductor.org/packages/2.8/data/ annotation/html/RmiR.hsa.html]

26. McVicker G, Green P: Genomic signatures of germline gene expression. Genome Res 2010, 20:1503.

27. Barberi T, Willis LM, Socci ND, Studer L: Derivation of multipotent mesenchymal precursors from human embryonic stem cells. PLoS Med 2005, 2:e161.

28. Chalmel F, Rolland AD, Niederhauser-Wiederkehr C, Chung SSW Demougin P, Gattiker A, Moore J, Patard JJ, Wolgemuth DJ, Jégou B: The conserved transcriptome in human and rodent male gametogenesis. Proc Natl Acad Sci USA 2007, 104:8346-8351

29. Ge X, Yamamoto S, Tsutsumi S, Midorikawa Y, Ihara S, Wang SM, Aburatani $\mathrm{H}$ : Interpreting expression profiles of cancers by genome-wide survey of breadth of expression in normal tissues. Genomics 2005, 86:127-141.

30. Houmard B, Small C, Yang L, Naluai-Cecchini T, Cheng E, Hassold T, Griswold M: Global gene expression in the human fetal testis and ovary. Biol Reprod 2009, 81:438-443.

31. Looijenga LHJ, Hersmus R, Gillis AJM, Pfundt R, Stoop HJ, van Gurp RJ, Veltman J, Beverloo HB, van Drunen E, Geurts van Kessel A: Genomic and expression profiling of human spermatocytic seminomas: primary spermatocyte as tumorigenic precursor and DMRT1 as candidate chromosome 9 gene. Cancer Res 2006, 66:290-302.

32. Kocabas AM, Crosby J, Ross PJ, Otu HH, Beyhan Z, Can H, Tam WL, Rosa GJM, Halgren RG, Lim B: The transcriptome of human oocytes. Proc Natl Acad Sci USA 2006, 103:14027-14032.

33. Korkola JE, Houldsworth J, Chadalavada RSV, Olshen AB, Dobrzynski D, Reuter VE, Bosl GJ, Chaganti R: Down-regulation of stem cell genes, including those in a 200-kb gene cluster at 12p13. 31, is associated with in vivo differentiation of human male germ cell tumors. Cancer Res 2006, 66:820-827.

34. Sato N, Sanjuan IM, Heke M, Uchida M, Naef F, Brivanlou AH: Molecular signature of human embryonic stem cells and its comparison with the mouse. Dev Biol 2003, 260:404-413.

35. Skottman H, Mikkola M, Lundin K, Olsson C, Strömberg AM, Tuuri T, Otonkoski T, Hovatta O, Lahesmaa R: Gene expression signatures of seven individual human embryonic stem cell lines. Stem Cells 2005, 23:1343-1356.

36. Su Al, Wiltshire T, Batalov S, Lapp H, Ching KA, Block D, Zhang J, Soden R, Hayakawa M, Kreiman G, et al: A gene atlas of the mouse and human protein-encoding transcriptomes. Proc Natl Acad Sci USA 2004, 101:6062-6067. 
37. Hubbell E, Liu WM, Mei R: Robust estimators for expression analysis. Bioinformatics 2002, 18:1585-1592.

38. BioGPS. [http://biogps.org].

39. Yang J, Su Al, Li WH: Gene expression evolves faster in narrowly than in broadly expressed mammalian genes. Mol Biol Evol 2005, 22:2113-2118.

40. The Cancer Gene Census database. [http://www.sanger.ac.uk/genetics/ CGP/Census/].

41. Volinia S, Mascellani N, Marchesini J, Veronese A, Ormondroyd E, Alder H, Palatini J, Negrini M, Croce CM: Genome wide identification of recessive cancer genes by combinatorial mutation analysis. PLoS One 2008, 3 e3380.

42. Xia J, Sun J, Jia P, Zhao Z: Do cancer proteins really interact strongly in the human protein-protein interaction network? Comput Biol Chem 2011, 35:121-125.

43. Chen FC, Chen CJ, Li WH, Chuang TJ: Human-specific insertions and deletions inferred from mammalian genome sequences. Genome Res 2007, 17:16-22.

44. 17-Way vertebrate alignment in the UCSC Genome Browser. [http:// hgdownload.cse.ucsc.edu/goldenPath/hg18/multiz17way/.

45. Deaton $\mathrm{AM}, \mathrm{Bird} \mathrm{A}: \mathrm{CpG}$ islands and the regulation of transcription. Genes Dev 2011, 25:1010-1022.

46. Illingworth RS, Gruenewald-Schneider U, Webb S, Kerr ARW, James KD, Turner DJ, Smith C, Harrison DJ, Andrews R, Bird AP: Orphan CpG islands identify numerous conserved promoters in the mammalian genome. PLOS Genet 2010, 6:e1001134.

47. Bird A: DNA methylation patterns and epigenetic memory. Genes Dev 2002, 16:6-21.

48. Walsh CP, Bestor TH: Cytosine methylation and mammalian development. Genes Dev 1999, 13:26-34

49. Warnecke PM, Clark SJ: DNA methylation profile of the mouse skeletal alpha-actin promoter during development and differentiation. Mol Cell Biol 1999, 19:164-172.

50. Wang $X$, El Naqa IM: Prediction of both conserved and nonconserved microRNA targets in animals. Bioinformatics 2008, 24:325-332.

51. Krek A, Grun D, Poy MN, Wolf R, Rosenberg L, Epstein EJ, MacMenamin P, da Piedade I, Gunsalus KC, Stoffel M: Combinatorial microRNA target predictions. Nat Genet 2005, 37:495-500.

52. Rajewsky N: microRNA target predictions in animals. Nat Genet. 2006, 38(Suppl:::S8-S13.

53. Cheng C, Bhardwaj N, Gerstein M: The relationship between the evolution of microRNA targets and the length of their UTRs. BMC Genomics 2009, 10:431.

54. Kim SH, Yi SV: Understanding relationship between sequence and functional evolution in yeast proteins. Genetica 2007, 131:151-156.

55. Eisenberg $E$, Levanon EY: Human housekeeping genes are compact. Trends Genet 2003, 19:362-365.

56. Stark A, Brennecke J, Bushati N, Russell RB, Cohen SM: Animal MicroRNAs confer robustness to gene expression and have a significant impact on 3'UTR evolution. Cell 2005, 123:1133-1146.

57. Cui Q, Yu Z, Pan Y, Purisima EO, Wang E: MicroRNAs preferentially target the genes with high transcriptional regulation complexity. Biochem Biophys Res Commun 2007, 352:733-738

58. Xie X, Lu J, Kulbokas E, Golub TR, Mootha V, Lindblad-Toh K, Lander ES, Kellis M: Systematic discovery of regulatory motifs in human promoters and 3 UTRs by comparison of several mammals. Nature 2005, 434:338-345.

59. PACdb. [http://harlequin.jax.org/pacdb/].

60. Thompson JD, Higgins DG, Gibson TJ: CLUSTAL W: improving the sensitivity of progressive multiple sequence alignment through sequence weighting, position-specific gap penalties and weight matrix choice. Nucleic Acids Res 1994, 22:4673-4680.

61. Kimura M: A simple method for estimating evolutionary rates of base substitutions through comparative studies of nucleotide sequences. $J$ Mol Evol 1980, 16:111-120.

62. Sun J, Zhao Z: A comparative study of cancer proteins in the human protein-protein interaction network. BMC Genomics 2010, 11(Suppl):S3-S5.

63. Gu X, Su Z, Huang Y: Simultaneous expansions of microRNAs and protein-coding genes by gene/genome duplications in early vertebrates. J Exp Zool B Mol Dev Evol 2009, 312B:164-170.
64. Heimberg AM, Sempere LF, Moy VN, Donoghue PC, Peterson KJ: MicroRNAs and the advent of vertebrate morphological complexity. Proc Natl Acad Sci USA 2008, 105:2946-2950.

65. Mandrioli M: A new synthesis in epigenetics: towards a unified function of DNA methylation from invertebrates to vertebrates. Cell Mol Life SC 2007, 64:2522-2524.

doi:10.1186/1471-2164-12-S5-S15

Cite this article as: Su et al:: Functional complementation between transcriptional methylation regulation and post-transcriptional microRNA regulation in the human genome. BMC Genomics 201112 (Suppl 5):S15.

\section{Submit your next manuscript to BioMed Central and take full advantage of:}

- Convenient online submission

- Thorough peer review

- No space constraints or color figure charges

- Immediate publication on acceptance

- Inclusion in PubMed, CAS, Scopus and Google Scholar

- Research which is freely available for redistribution

Submit your manuscript at www.biomedcentral.com/submit
Ciomed Central 